$N$-Benzyl-tetrahydrochinolin

entsteht durch $2 \frac{1}{2}$-stündiges Erwärmen von $26.5 \mathrm{~g}$ Tetrahydrochinolin and $25.3 \mathrm{~g}$ Benzylchlorid auf $100^{\circ}$. Nach der üblichen Aufarbeitung erhält man bei der Destillation unter vermindertem Druck (46 mm) eine von $215-225^{\circ}$ siedende Hauptfraction, die nach abermaliger Rectification unter $38 \mathrm{~mm}$ Druck von $218-222^{\circ}$ siedet. Das so gewonjene, dickflüssige Oel erstarrt beim Stehen in der Kälte zu fächerförmig angeordneten Nadeln, welche nach wiederholter Krystallisation aus absolutem Alkohol farblos werden und den Schmp. 36-37 zeigen.

0.1106 g Sbst.: $0.3466 \mathrm{~g} \mathrm{CO}_{2}, 0.476 \mathrm{~g} \mathrm{H}_{2} \mathrm{O}$.

$$
\begin{aligned}
& \mathrm{C}_{16} \mathrm{H}_{17} \mathrm{~N} \text {. Ber. C 86.09, H 7.62. } \\
& \text { Gef. » 85.71, 》 7.81. }
\end{aligned}
$$

Die Ausbeuten an dieser tertiären Base sind sehr schlecht; $N$-Benzyltetrahydrochinolin verbindet sich selbst mit Allyljodid nur sehr langsam.

Die Untersuchung über die Einwirkung von Jodessigsäureestern auf Kairolin (s. o.) wird in Gemeinschaft mit Hrn. R. Oechslen fortgesetzt.

Tübingen, im December 1901.

34. Carl Bülow und Georg List: Ueber die relative Fasicitätsdifferenz der beiden Amidogruppən substituirter Diamine.

II. o-Toluylendiamin $\left[\mathrm{CH}_{3}: \mathrm{NH}_{2}: \mathrm{NH}_{2}=1: 3: 4\right]$.

[Mițheilung aus dem Laborat. des chem. Instituts der Universität Täbingen.] (Eingeg. am 30. Dee. 1901; mitgeth. in der Sitzung von Hrn. C. Neuberg.)

Vor nicht allzulanger Zeit hat C. Bülow ${ }^{1}$ ) gezeigt, dass die beiden Amidogruppendes (unsymm.) m-Toluylendiamins, $\mathrm{CH}_{3}$ : $\mathrm{NH}_{2}: \mathrm{NH}_{2}=1: 2: 4$ in Bezug auf ihre Basicität nicht gleichwerthig sind, sondern dass das dem Methyl gegenüberstehende (para-)A mid reactionsfähiger ist als das benachbarte orthoständige. Der Beweis gipfelte in der Voraussetzung, dass die von beiden stärker basische Amidogruppe auch die grössere Neigung zeigen würde, sich mit einer molekularen Menge von Diacetbernsteinsïureester zum Pyrrolderivat zu kuppeln, als die

1) Bülow, diese Berichte 33, 2364 [1900]. 
schwäeher basische. Das experimentell gewonnene, hier vorangestellte Endresultat der Untersuchung liess sich in allerbesten Einklang mit einer Beobachtung Tiemann's ${ }^{1}$ ) bringen, derzufolge ein Mol.-Gewicht $m$-Toluylendiamin durch längeres Kochen mit etwas weniger als zwei Mol.-Gewichten Eisessig, dem eine geringe Menge Wasser zugesetzt worden war, ganz überwiegend das in paraStellung monoacetylirte Product lieferte. Und endich gab ein dritter Versuch ein ebenfalls nur durch Basicitätsdifferenz zu erklärendes Resultat: behandelt man das diacetylirte (unsymm.) m-Toluylendiamin in wässrig-alkoholischer Lösung mit einem Mol.-Gewicht Aetznatron, so erhält man als einzig fassbares monoacetylirtes Diamin das Tiemann'sche Product; es spaltet sich eben naturgemäss der weniger stark haftende Essigsäurerest zuerst ab.

Damit war bewiesen worden, dass das Verfahren, kernsubstituirte Diamine mit Diacetbernsteinsäureester zu Pyrrolabkömmlingen zu condensiren, sich besonders dann eignet die Frage über die Basicitätsdifferenzen der Amidogruppen zweisäuriger Basen zur Entscheidung zu bringen, wenn es gelingt, die Constitution des Kuppelungsproductes einwandfrei $z u$ formuliren.

Wir haben uns zunächst etwas ausfäbrlicher mit dem. unsymmetrischen $o$ - Toluylendiamin, $\left[\mathrm{CH}_{3}: \mathrm{NH}_{2}: \mathrm{NH}_{2}=1: 3: 4\right]$, beschätigt und gefunden, dass auch in ihm die dem Methyl gegenüberstehende para-Amidogruppestärker basisch ist als diejenige, welche sich in Metastellung befindet.

Die Methoden der Monoacetylirung des Diamins, oder die der Elimination des einen Essigsäurerestes aus dem 1.3.4-Diacetyltoluylendiamin konnten hier nicht angewandt werden, da die Base bei der Behandlung mit Eisessig, und selbst dann, wenn man das Nitro-acet$p$-toluid, $\left[\mathrm{CH}_{3}: \mathrm{NO}_{2}: \mathrm{NH}=1: 3: 4\right]$, unter den geeignetsten Bedingungen reducirt, nicht in die gewünschten Verbindungen, sondern in das Dimetbylbenzimidazol (o-Toluylenacetamidin) von der Formel

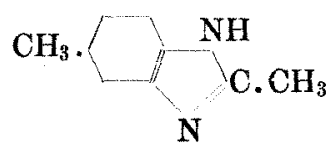

äbergeht.

Auch erschien es von vornherein fraglich, ob 1.2-Diamine mit 1.4-Diketonen überhaupt zu Pyrrolderiraten condensirbar seien, oder ob nicht etwa anderweitige, unvorhergesehene Körper gebildet würden. Es hat sich aber ergeben, dass das o-Toluylendiamin grosse Neigung zeigt, mit $z$ wei Molekeln Diacetbernsteinsäureester zu rea-

1) Tiemano, diese Berichte 3, 221 [1870]. 
giren und den Toluylendis-[2.5-dimethylpyrrol-3.4-dicarbonsäureester] von der Formel

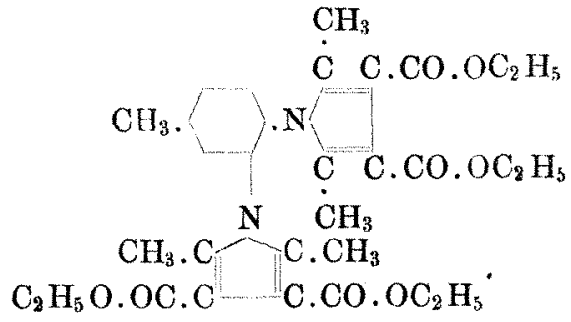

zu bilden. Unter anders gewählten Bedingungen gelingt es indessen ziemlich leicht, beide Componenten in äquimolekularen Mengen zu vereinigen, wobei dann, je nach der relativen Stärke der Amidogruppen, entweder die Verbindung

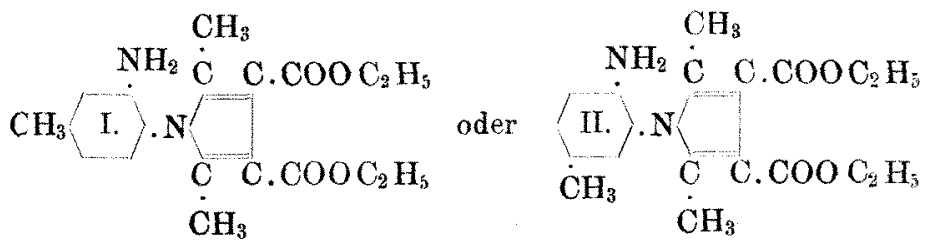

oder aber beide zusammen entstehen müssten. Letztere Möglichkeit trat nicht ein, da es nicht gelang, aus der Reactionsflüssigkeit mehr als ein einziges Pyrrolderivat mit freier Amidogruppe zu isoliren. Die endgültige Entscheidung über die wirkliche Zusammensetzung, d. h. die Entscheidnng zwischen Formel I und II, konnte durch Entamidirung geführt werden: Amidotolyl-[2.5-dimethylpyrrol-3.4-dicarbonsäureester] wurde diazotirt, die gewonnene Diazoverbindung zum Hydrazia reducirt und endlich die. NH. $\mathrm{NH}_{2}$ - Gruppe nach bekannter Methode durch Wasserstoff ersetzt. Der auf diese Weise erhaltene Tolyl-2.5. dimethylpyrrol-3.4-dicarbonsäureester musste dann identisch sein mit dem aus $p$-Toluidin oder mit dem aus $m$-Toluidin nnd Diacetbernsteinsäureester entstehenden Pyrrolabkömmling.

Da sich nun herausstellte, dass das Entamidirungsproduct mit dem Knorr'schen 1) N-p-Tolyl-2.5-dimethyl-pyrrol-3.4-dicarbonsäureester, sein Verseifungsproduct mit der entsprechenden Säure und sein ans ihm entstehendes Tolyldimethylpyrrol mit der Knorr'schen Base völlig übereinstimmten, so ist $u n z w e i f e l h a f t$ erwiesen, dass sich bei der äquimolekularen Condensation des unsymmetrischen o-Toluylendiamins mit Diacetbernsteinsäureester nur die paraständige Amidogruppe, als die relativ stärkere von beiden, betheiligt.

1) Knorr, diese Berichte 18, 299 [1885]. 
Experimenteller Theil.

n-Toluylen-dis-[2.5-dimethylpyrrol-3.4-dicarbonsäure].

$4 \mathrm{~g}$ o-Tolnylendiaminchlorhydrat (1 Mol.) and $10.4 \mathrm{~g}$ Diacetbernsteinsäureester (2 Mol.) wurden in einem Gemisch von $40 \mathrm{ccm}$ Alkohol mit $40 \mathrm{cem}$ Eisessig gelöst, und das Ganze zunächst 2 Stunden am Rückflusskühler gekocht. Dann verdünnt man mit $20 \mathrm{ccm}$ Wasser and erhitzt nochmals 2 Stunden zum Sieden. Die Salzsäure war durch Zusatz von $3 \mathrm{~g}$ Natrinmacetat abgestumpft worden. Beim Erkalten schied sich der Ester harzig aus. Er wurde in Aether aufgenommen, die ätherische Lösung zuerst mit stark verdünnter Natronlange, dann mit 1-procentiger Salzsäure gewaschen, getrocknet und der Aether abgedunstet. Da der Rückstand durchans keine Neigung zum Krystallisiren zeigte, so wurde er in $50 \mathrm{cem}$ Methylalkohol gelöst, $15 \mathrm{~g}$ festes Aetzkali hinzugegeben und die alkalische Flüssigkeit auf dem Wasserbade erwärmt. Nach Verlauf von etwa 15 Minuten beginnt das Kaliumsalz der Säure, sich in feinen Krystallen abzuscheiden. Man verdampft den Alkohol, verdünnt mit dem 4-fachen Volumen Wasser und versetzt zunächst mit Essigsäure bis zur sauren Reaction, um nicht völlig verseiften Ester abzuscheiden, lässt dann einige Stunden in der Kälte stehen, filtrirt von der in geringer Menge entstrndenen Ausscheidung ab und übersättigt endlich mit Salzsäure. Sofort scheidet sich die Tetracarbonsäure als ein dicker voluminöser Niederschlag ans. Man sangt ihn ab, wäscht ihn mit kaltem Wasser gut aus, löst ihn in möglichst wenig verdũnnter Sodalauge, übersättigt die Flüssigkeit zunächst wiederum mit Essigsäure, filtrirt und fällt die nun reine Tetracarbonsäure durch Mineralsäure aus. Sie wurde aus verdünntem Alkohol umkrystallisirt. Ausbeute an reiner Sub$\operatorname{stan} z: 4 \mathrm{~g}$.

o-Toluylen-dis-[dimethylpyrroldicarbonsäure] schmilzt unter Zersetzurig bei $272^{\circ}$. Von den anderen, gebräuchlichen, organischen Lösungsmitteln wird sie nicht oder nur schwer aufgenommen.

$0.1212 \mathrm{~g}$ Sbst.: $0.2702 \mathrm{~g} \mathrm{CO}_{2}, 0.0532 \mathrm{~g} \mathrm{H}_{2} \mathrm{O} .-0.1589 \mathrm{~g}$ Sbst.: $0.3403 \mathrm{~g}$ $\mathrm{CO}_{2}, 0.0692 \mathrm{~g} \mathrm{H}_{2} \mathrm{O} .-0.1589 \mathrm{~g}$ Sbst.: $9 \mathrm{~cm} \mathrm{~N}(190,728 \mathrm{~mm})$.

$$
\begin{aligned}
& \mathrm{C}_{23} \mathrm{H}_{22} \mathrm{O}_{8} \mathrm{~N}_{2} \text {. } \begin{array}{l}
\text { Ber. C } 60.78, \quad \mathrm{H} 4.98, \\
\text { Gef. } \gg 60.80,60.45, ~
\end{array} 4.87,5.2, 》 6.17 .
\end{aligned}
$$

Das eben beschriebene Dispyrrolderivat bildet sich auch, wenn man aequimolekulare Mengen Diamin und Diacetbernsteinsäureester 4-5 Stunden in essigsaurer Lösung am Rückflusskühler kocht; die Ausbeute ist in diesem Falle allerdings wenig befriedigend. $\mathrm{Da}$ es aber überhaupt trotz dieser nicht gerade sebr günstigen Bedingungen entsteht, so muss man folgern, dass auch die metaständige Amidogruppe eine an sich noch immerhin recht bemerkenswerthe Basicität besitzt. Damit steht in Einklang, dass das o-Toluylendiamin zweisäurige Salze bildet. 
Das saure Silbersalz der o-Toluylen-dis-[dimethylpyrroldicarbansäure] von der empirischen Formel $\mathrm{C}_{23} \mathrm{H}_{20} \mathrm{O}_{8} \mathrm{~N}_{2} \mathrm{Ag}_{2}$ wird aus dem ihm entsprechenden sauren Ammoniumsalz gewonnen. Letzteres bildet sich, wenn man die Tetracarbonsäure in überschüssigem Ammoniak löst und die Flüssigkeit so lange zum Sieden erhitzt, bis der Geruch nach Ammoniak vollkommen verschwunden ist. Versetzi man dann mit Silbernitratlösung, so fällt das Silbersalz als dichter, weisser, ziemlich lichtbeständiger Niederschlag ans, der gut und andauernd mit lauwarmem Wasser ansgewaschen wird. Er löst sich unverändert in verdünnter Ammoniakflüssigkeit, wird aber beim Erwärmen mit schwacher Natronlauge unter Silberhydroxydausscheidung in das Natriumsalz umgesetzt. Aus dem Filtrat kann man dureh Zusatz von Salzsäure die unveränderte Tetracarbonsäure vom Schmp. $272^{6}$ ausfällen.

\section{$0.2458 \mathrm{~g}$ Silbersalz: $0.0806 \mathrm{~g} \mathrm{Ag}$.}

$$
\mathrm{C}_{23} \mathrm{H}_{20} \mathrm{O}_{8} \mathrm{~N}_{2} \mathrm{Ag}_{2} \text {. Ber. Ag 32.5, Gef. Ag 32.8. }
$$

Der etwas $z \mathfrak{n}$ hoch gefundene Silbergehalt dürfte dadurch za erklären sein, dass eine der beiden schwächeren Carboxylgruppen welche sich, wie die beiden anderen stärkeren, beim Uebersättigen mit Ammoniak zuerst anch an der Salzbildung betheiligten, beim darauf folgenden Kochen dann ihr Ammoniak nicht vollständig durch Dissociation verloren hat.

Bereits von Knorr (a. a. O.) wurde beobachtet, dass sich aus Pyrrol-o-dicarbonsäuren saure Ammoniumsalze auf die angegebene Weise gewinnen lassen. Da nun in unserem Falle z wei Pyrrol-3.4-diearbonsäurereste an einem Toluylenkern hängen, so ist anzunehmen, dass die beiden Metallatome unseres Silbersalzes auch anf beide Reste gleichmässig vertheilt sind. Die beiden freien Carboxylgruppen dürften in ihrer relativen Acidität noch hinter derjenigen des Pheuolphtaleins zurückstehen; denn titrirt man die o-Toluylen-dis-[dimethyl-3.4-dicarbonsäure] mit $1 / 10^{-n} n$-Kalilauge und wählt jene Substan $z$ als Indicator, so erhält man Resultate, die aunähernd auf eine Dicarbonsäure, aber durchaus nicht auf eine Tetracarbonsäure stimmen.

$0.2072 \mathrm{~g}$ Sbst. verbranchen $9.5 \mathrm{ccm} 1 / \mathrm{to}_{-}-n_{\text {. }}$ KOH. - $0.1641 \mathrm{~g}$ Sbst. verbraxchen $7.4 \mathrm{ecm} 1 / 10^{-12}$ - $\mathrm{KOH}$.

Ber. für die Tetracarbonsănre: $\mathrm{KOH} 49.3 \mathrm{pCt}$, thatsächlich sind verbrancht worden an $\mathrm{KOH} 25.6$ und $25.2 \mathrm{pCt}$.

Es wurde demnach etwa nur die Hälfte des theoretisch zum $A b$ sittigen von vier COOH-Gruppen nothwendigen Alkalilauge verwand, als die weiter hinzugefügte Kalilauge bereits auf das in Lösung befindliche Phenolphtaleïn zu wirkeu begamn. 
A midotolyl-2.5-dimethylpyrroldicarbonsäure.

Man löst $4 \mathrm{~g} o$-Toluylendiaminchlorhydrat $(1 \mathrm{Mol}$.$) und 5.2 \mathrm{~g}$ Diacetbernsteinsäureester $(1 \mathrm{Mol}$.) in $25 \mathrm{ecm}$ Eisessig auf, fügt $20 \mathrm{~cm}$ Alkohol hinzu, verdünnt mit $10 \mathrm{~cm}$ Wasser und kocht eine halbe Stunde am Rückflusskühler. Dann giebt man $20 \mathrm{ccm}$ Wasser zur Lösung, erhitzt nochmals eine Stunde zum Sieden und lässt erkalten. Die nach längerem Stehen im Eisschrank ausgeschiedene harzige Masse zeigt wenig Neigung zum Krystallisiren. Sie wird in Aether aufgenommen, die ätherische Solution zunächst mehrere Male mit reinem Wasser tüchtig gewaschen und zuletzt das Waschwasser durch einige Tropfen Salzsäure angesäuert. Dann schüttelt man sie gehörig mit sehr stark verdünnter Natronlauge durch und verjagt endlich das Lösungs̀mittel. Der hinterbleibende Rückstand wird sofort auf die Säure weiter verarbeitet, indem man ihn mit $50 \mathrm{ccm}$ Methylalkohol übergiesst, $10 \mathrm{~g}$ Aetzkali hinzufügt und das Ganze zum Sieden erhitzt. Nach einiger Zeit beginnt das Kaliumsalz der Dicarbonsäure sich auszuscheiden. Man verdampft den überschüssigen Alkohol auf dem Wasserbade und erhält nun beim Verdünnen mit etwa der vierfachen Menge Wasser eine klare Lösung, aus der sich durch Uebersättigen mit Essigsäure nichts abscheidet, oder aber nur minimale Mengen Substanz ausgefällt werden. Unter allen Umständen lässt man längere Zeit in der Kälte stehen, filtrirt eventuell und fügt nun nur eben so viel Salzsäure hinzu, bis ein weiterer Niederschlag nicht entsteht. Die Amidotolyldimethylpyrroldicarbonsäure scheidet sich dabei als voluminöser Niedersehlag ab. Sie wird nach dem Absaugen und Waschen durch verdünnte Natriumcarbonat-Lösung aufgenommen und, wie angegeben, wieder ausgefällt. Die gewonnene Dicarbonsäure ist durchaus einheitlich, sie schmilzt unter Zersetzung bei $205^{\circ}$, wird von verdünntem Alkohol ziemlich gut gelöst und krystallisirt beim Erkalten in feinen Nädelchen aus. In Aether, Benzol, Nitrobenzol und anderen Solventien ist sie nicht gut löslich.

Das Vorhandensein der Amidogruppe lässt sich leicht erweisen, indem man die Säure in Wasser suspendirt, sie vorsichtig durch eben hinreichenden Zusatz von Sodalauge in Lösung bringt, genügend Salzsäure hinzufügt und dann den feinen Brei mit Natriumnitrit versetzt. Das Diazoniumsalz ist löslich. Fügt man die klar gewordene Flüssigkeit zu einer alkalischen Lösung von R-Salz, so scheidet sich sofort ein ziegelrother Azofarbstoff aus, der beim Kochen mit Wasser eine bläulich-rothe Solution giebt. Resultat:

Wine Stickstoffbestimmung der Dicarbonsäure ergab folgendes

$0.1178 \mathrm{~g}$ Sbst.: $10.2 \mathrm{ccm} \mathrm{N}\left(17^{\circ}, 739 \mathrm{~mm}\right)$.

$\mathrm{C}_{15} \mathrm{H}_{16} \mathrm{O}_{4} \mathrm{~N}_{2}$. Ber. N 9.75. Gef. N 9.9 . 
Das saure Ammoniumsala ${ }^{1}$ der Amidotolyl-2.5-dimethylpyrrol-3.4-dicarbonsäure von der Formel $\mathrm{C}_{15} \mathrm{H}_{15} \mathrm{O}_{4} \mathrm{~N}_{2} \cdot \mathrm{NH}_{4}$ warde nach der bekannten Methode gewonnen und aus ihm durch Umsetzung mit Silbernitrat das saure Silbersalz der Dicarbonsäure hergestellt. Letzteres ist in heissem Wasser ziemlich schwer löslich, wird aber leicbt von Ammoniak aufgenommen.

Analyse des sauren Silbersalzes der Dicarbonsäure:

$0.2400 \mathrm{~g}$ Silbersalz hinterliessen nach dem Glühen: $0.0648 \mathrm{~g} \mathrm{Ag}$. $\mathrm{C}_{15} \mathrm{H}_{15} \mathrm{O}_{4} \mathrm{~N}_{2}$. Ag. Ber. Ag 27.3. Gef. Ag 27.00.

Constitutions beweis

für die Amidotolyl-2.5-dimethylpyrrol-3.4-dicarbonsäure.

Wie bereits in der Einleitung erwähat warde, lässt sich die genannte Amidosäure durch Entamidirung in die Knorr'sche p-Tolyl2.5-dimethylpyrrol-3.4-dicarbonsäure (Ber. 18, 307 [1885]) überführen. Die paraständige A midogruppe des unsymmetrischen o-Toluylendiamins hat also ihr Stickstoffatom zur Bildung des Pyrrolringes hergegeben.

Die Elimination der Amidogruppe wurde folgendermaassen bewerkstelligt:

1. $10 \mathrm{~g}$ Amidotolyl-2.5-dimethyl-3.4-dicarbonsäure wurden mit $30 \mathrm{~cm} 18$-procentiger Salzsäure zu einem Brei verrührt, der dann unter guter Kühlung so lange mit gasförmiger »salpetriger Säures behandelt wurde, bis alles klar in Lösung gegangen war. Diese Lösung liess man aus einem Tropftrichter langsam in $250 \mathrm{ccm}$ kochenden, absoluten Alkohols einfliessen und erhitzte das Ganze auf dem Wasserbade noch so lange weiter zum Sieden, bis die Stickstoffentwickelung aufgehört hatte. Nach Zusatz von $100 \mathrm{ccm}$ Wasser wurden der Alkohol, Aldehyd und sonstige flüchtige Zersetzangsproducte mit Wasserdämpfen übergetrieben und der im Destillationskolben zurückgebliebene, wässrige, saure Rest, nach der Filtration, auf dem Wasserbade au etwa ein Viertel seines Volumens eingedampft. Man macht dann mit Soda eben alkalisch, filtrirt wiederum und fällt die $p$-Tolyldimethylpyrroldicarbonsäure mit Salzsäure aus. Nach nochmaligem Umlösen, Wiederausfällen und Umkrystallisiren aus verdünntem Alkohol blieb der Schmelzpunkt constant bei $249-250^{\circ}$. Ausbeute nur $1.5 \mathrm{~g}$.

2. $10 \mathrm{~g}$ Amidotolyl-2.5-dimethylpyrrol-3.4-dicarbonsäure wurden wie oben diazotirt und dann nach der bekannten $\mathrm{E}$. Fischer'schen Methode in das Hydrazin verwandelt. Aus dem nicht besonders isolirten Hydrazin, welches alle charakteristischen Reactionen dieser Körperklasse zeigte, konnte durch Kochen mit Kupfersulfat- oder Eisenchlorid-

1) Toh beabsichtige, die relative Aciditätsdifferenz der COOH-Gruppen mebrwerthiger substituirter Carbonsâuren genauer zu untersuchen. Bülow. 


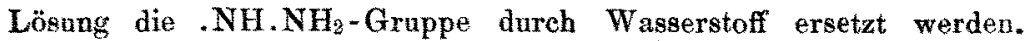
Aethert man nun die Reactionsflüssigkeit verschiedene Male aus und verdampft das Lösungsmittel, so hinterbleibt die $\mathrm{Knorr}$ 'sche Säure, die nach einmaligem Umkrystallisiren aus verdünntem Alkohol sofort den richtigen Schmp. $250^{\circ}$ zeigt.

0.2501 g Sbst.: $12.2 \mathrm{cem} \mathrm{N}\left(20^{\circ}, 730 \mathrm{~mm}\right)$,

$$
\mathrm{C}_{15} \mathrm{H}_{15} \mathrm{O}_{4} \mathrm{~N} \text {. Ber. N 5.15. Gef. N 5.3. }
$$

Aus dieser Säure wurde durch Destillation bei $694 \mathrm{~mm}$ Druck, unter Abspaltung von Kohlensäure, das Knorr'sche p-Tolyldimethylpyrrol als farbloses, bald krystallinisch erstarrendes Oel erbalten. Es besitzt, nach dem Umkrystallisiren aus Aether, den Schmp. $45-46^{\circ}$, ist unlöslich in Wasser, Alkalien und verdünnten Säuren, leicht flüchtig mit Wasserdämpfen und zeigt in ausgezeichneter Weise die Pyrrolreaction.

$0.1357 \mathrm{~g}$ Sbst.: $8.6 \mathrm{ccm} \mathrm{N}\left(15^{\circ}, 740 \mathrm{~mm}\right)$.

$$
\mathrm{C}_{13} \mathrm{H}_{15} \text { N. Ber. N 7.6. Gef. N 7.33. }
$$

Wir sind damit beschäftigt, die relative Basicität der Amidogruppen des unsymmetrisehen (v-) o-Toluylendiamins $\left[\mathrm{CH}_{3}: \mathrm{NH}_{2}: \mathrm{N} \mathrm{H}_{2}=\right.$ $1: 2: 3]$ und des $p$-Tolnylendiamins $\left[\mathrm{CH}_{3}: \mathrm{NH}_{2}: \mathrm{NH}_{2}=1: 2: 5\right] \mathrm{zu}$ untersuchen, um zu sehen, ob das vicinale und das unsymmetrische, orthoständige, oder ob das metaständige Amid das chemisch actirere sei.

\section{A. Pinner und R. Schwarz: Ueber Pilocarpin.}

(Eingegangan am 27. December 1901; rorgetragen in der Sitzung von Hrn.

\section{A. Pinner.)}

Im Decemberheft des Journal of the Chemical Society $(79,1331)$ veröffentlicht $\mathrm{Hr}$. Jowett eine dritte Mitheilnng seiner Untersuchungen süber die Constitution des Pilocarpins«. Hr. Jowett hat von Anfang an nicht das Pilocarpin selbst, sondern das daraus durch Erhitzen des Chlorhydrats oder durch Einwirkung von Alkalien entstehende isomere Alkaloïd, das Isopilocarpin, zum Gegenstand seiner Forschungen gemacht. In seiner ersten Abhandlung ${ }^{1}$ ) theilte er u. a. mit, dass er das salpetersaure Isopilocarpin bei $80^{\circ}$ mit 6 Mol. Permanganat oxydirt habe, sodass also neun Atome Sauerstoff verbraucht wurden, und dass er hierbei, neben Essigsäare, eine lactonartige Säure $\mathrm{C}_{7} \mathrm{H}_{10} \mathrm{O}_{4}$ zu $70 \mathrm{pCt}$. der berechneten Menge erhalten habe. Isolirt habe er die Sänre, indem er die alkoholische Lösung der Kaliumsalze mittels Salzsäuregas esterificirt und das im Vacunm rectificirte, bei gewöhnlichem Druck fast constant (nämlich zu 90 pCt.) bei $299^{n}$ siedende Oel, ans dessen Analyse er die Forme!

1) Jowrm. Chem. Soe. 77, 851. 